encroachment across $5^{\prime}$ or $3^{\prime} \mathrm{CpG}$ island borders. $\mathrm{CpG}$ island methylation encroachment is a widespread phenomenon in clinical prostate and breast cancer and is commonly associated with gene suppression. By integrating whole-genome DNA methylation sequencing and chromatin modification sequencing data, we have revealed that the pattern of histone lysine 4 monomethylated (H3K4me1) nucleosomes in normal cells is associated with the different modes of $\mathrm{CpG}$ island hypermethylation. Notably, we show that genetic manipulation of lysine histone methyltransferase 2D (KMT2D) results in concordant alterations in $\mathrm{H} 3 \mathrm{~K} 4 \mathrm{me} 1$ levels and corresponding $\mathrm{CpG}$ island border DNA methylation encroachment. Prostate cancer DNA harbouring KMT2D mutations also display reduced erosion of $\mathrm{CpG}$ island border methylation. Our findings establish a unique role for $\mathrm{H} 3 \mathrm{~K} 4 \mathrm{me} 1$ in the demarcation of $\mathrm{CpG}$ island borders in normal cells, which become eroded in cancer. ${ }^{1}$

Reference

1. Skvortsova K, Masle-Farquhar E, Luu P-L, et al. DNA

hypermethylation encroachment at $\mathrm{CpG}$ island borders in cancer is predisposed by $\mathrm{H} 3 \mathrm{~K} 4$ monomethylation patterns. Cancer Cell 2019; 35: 297-314.e8.

\section{MOLECULAR PROFILING IN HAEMATOLOGICAL MALIGNANCIES - THE MOST-LLY STUDY}

\author{
Steven Lane ${ }^{1,2}$ \\ ${ }^{1}$ QIMR Berghofer, Gordon and Jessie Gilmour Leukaemia \\ Research Laboratory, QIMR Berghofer, Herston, Qld, \\ Australia; and ${ }^{2}$ Royal Brisbane and Women's Hospital, \\ Herston, Qld, Australia
}

MoST-LLy (Molecular Screening and Therapeutics in Leukaemia and Lymphoma) has a unique study design with molecular screening using Illumina's TruSight ${ }^{\mathrm{TM}}$ Oncology 500 Next Generation Sequencing (NGS) assay, and linking patients with advanced haematological malignancy with no standard of care treatment, to access targeted clinical trials and treatments they would otherwise not have access to.

The overarching MoST framework protocol governs molecular screening with each substudy reviewed and approved as an addenda to the program. We have developed two novel haematology specific treatment substudies with pharma that will soon open to recruitment, including:

- MoST 15: durvalumab + acalabrutinib for high-grade B cell lymphoma.

- MoST 16: PARP inhibitor for relapsed/refractory myeloid haematological malignancy.

MoST-LLy is the first molecular screening of its kind in blood cancers in Australia, and is being piloted across two sites initially with funding provided by the Leukemia Foundation of Australia and Tour de Cure to screen 240 patients, with plans to roll out nationally.

\section{GENETIC TESTING FOR FAMILIAL HYPERCHOLESTEROLAEMIA}

John R. Burnett $^{1,2}$

${ }^{1}$ Department of Clinical Biochemistry, PathWest Laboratory Medicine, Royal Perth Hospital and Fiona Stanley Hospital Network, Perth, WA, Australia; and ${ }^{2}$ School of Medicine, University of Western Australia, Nedlands, WA, Australia
Familial hypercholesterolaemia $(\mathrm{FH})$ is a codominant genetic disorder causing premature atherosclerotic cardiovascular disease (ASCVD). It is common, affecting 1 in 250 individuals worldwide and of the $\sim 100,000$ Australians estimated to have $\mathrm{FH}$, most are undiagnosed and treatment is often suboptimal. Several scoring algorithms based on low-density lipoproteincholesterol (LDL-C) levels, physical findings, and elements of personal and family history, help clinicians diagnose $\mathrm{FH}$ at varying levels of confidence ranging from possible to definite. Targeted DNA sequencing to identify the underlying pathogenic variant in any of the three $\mathrm{FH}$ genes ( $L D L R, A P O B$, and PCSK9) has become more accessible, but it is not essential for diagnosis of $\mathrm{FH}$, and is currently not widely used. However, identification of a pathogenic variant is considered the 'gold standard' for FH diagnosis. Expanded FH panels can identify pathogenic variants in other hypercholesterolaemia genes, such as APOE, LIPA, and $A B C G 5 / 8$. FH cascade screening is recommended to identify affected family members, and the benefits of early intervention to lower LDL-C and reduce the associated ASCVD burden are clear. National registries can also play a key role in identifying patients with FH. FH genetic testing should be incorporated as standard of care for patients and their relatives with suspected $\mathrm{FH}$

\section{THE ONCOGENE AND COAGULOME}

\author{
Kate Burbury $^{1,2}$ \\ ${ }^{I}$ Department of Haematology, Peter MacCallum Cancer \\ Centre, Melbourne Health, Vic, Australia; and ${ }^{2}$ University of \\ Melbourne, Vic, Australia
}

Cancer is the second-leading cause of death globally, predominantly due to metastasis - the escape of tumour cells from their initial to a distant site. Importantly, thromboembolism associated with cancer is the second-leading cause of cancer mortality, and in non-fatal events, a major contributor to morbidity, interruption to therapy and healthcare resource utilisation. Adding to the complexity, patients with cancer are also at high risk of haemorrhagic events during the course of their disease and treatment. Further understanding of haemostatic dysregulation is a significant unmet need in oncology. The pathogenesis is complex and involves the interplay of multiple factors. However, there is growing evidence that interactions between malignancies and the coagulation system are not random but represent coordinated and clinicallysignificant adaptations that enhance tumour cell survival, proliferation and metastatic potential. Hence understanding the interactions between haemostatic systems, vascular stroma and metastasis may not only provide insight into strategies that could potentially reduce the incidence of thrombohaemorrhagic events, but also help design strategies that are capable of modifying tumour biology and metastatic potential, and thereby improve overall survival. This presentation will provide an overview of biological and clinical evidence linking oncogenic signalling and coagulation phenotypes, and the opportunity for impact.

\section{MATCHING TARGET TO THERAPY IN CHILDHOOD CANCERS}

\section{Paul Ekert}

Translational Tumor Biology, Children's Cancer Institute, Randwick, NSW, Australia 
The Zero Childhood Cancer Program (ZERO) is Australia's first national paediatric cancer precision medicine program, focused on real-time recruitment and analysis of patients with high-risk paediatric cancer. The primary aim is a systematic evaluation of the feasibility and utility of a comprehensive molecular profiling platform consisting of germline and tumour WGS, RNAseq and CNS methylome analysis to identify clinically significant variants relevant to the biology of the tumour, diagnosis, clinical management or prognosis. Programs such as ZERO extend well beyond established gene-drug indications, and explore many novel aspects of cancer biology. One of the significant challenges faced in new models of personalised medicine is distilling which of the millions of molecular changes within a given tumour are pathogenic and relevant to clinicians. Novel variants are frequently identified which likely have pathogenic roles in cancers, but remain unsupported by functional validation studies. The therapeutic 'actionability' of such variants is a particularly fluid designation, being influenced by the availability of preclinical data, drugs and clinical trials.

\section{MACKENZIE'S MISSION}

Edwin P. Kirk $^{1,2,3}$

${ }^{1}$ Randwick Genomics Laboratory, New South Wales Health Pathology, Randwick, NSW, Australia; ${ }^{2}$ Centre for Clinical Genetics, Sydney Children's Hospital, Randwick, NSW, Australia; and ${ }^{3}$ School of Women's and Children's Health, UNSW, Randwick, NSW, Australia

Mackenzie's Mission, the Australian Reproductive Carrier Screening Project, is a research project funded by the Medical Research Future Fund that aims to screen 10,000 couples for 1300 genes associated with $>700$ genes. The project is studying uptake, proportion of couples found to be at high risk, reproductive decisions made by those couples and psychosocial, health economic, ethics and implementation research related to carrier screening. Despite some pandemic-related hitches, the project is progressing well; preliminary data will be presented.

\section{IS AUTOMATED GENOMIC REPORTING READY FOR PRIME TIME?}

Leslie Burnett $^{1,2,3}$

${ }^{1}$ Kinghorn Centre for Clinical Genomics, Garvan Institute of Medical Research, Darlinghurst, NSW, Australia; ${ }^{2}$ St Vincent's Clinical School, UNSW Sydney, Darlinghurst, NSW, Australia; and ${ }^{3}$ Sydney Medical School, University of Sydney, Sydney, NSW, Australia

The process of reducing the large genomic datasets obtained from sequencing a genome, to a smaller and more manageable filtered set of variants for analysis, is already usually an automated pipelined process. Similarly, many laboratory and bioinformatic workflows are already amenable to basic laboratory automation.

To explore taking genomic reporting to the next stage, it is possible to utilise more powerful bioinformatic tools. These can include linear programming, large-data science, machine learning and deep learning/artificial intelligence. While some of these techniques have sophisticated mathematical underpinnings, once developed, they are surprisingly easy to apply and use.

Examples will be given of the application of these tools to clinical problems. Their diagnostic performance already exceeds the capabilities of human experts, their speed similarly outperforms humans, and the reproducibility and consistency (and ease of use) of this approach strongly contributes to reduction in variability and resultant improvement in quality. Further applications include real-time point-of-care genomic diagnosis, and automated background reanalysis of unsolved cases. 